\title{
Limitações da cardiomioplastia no tratamento das cardiomiopatias
}

\author{
Luiz Felipe P. MOREIRA*, Edimar A. BOCCHI*, Noedir A. G. STOLF*, Pedro SEFERIAN Jr. *, Paulo M. \\ PÊGO - FERNANDES*, Antônio Carlos PEREIRA-BARRETO*, Fúlvio PILEGGI, Adib D. JATENE*
}

MOREIRA, L. F. P.; BOCCHI, E. A.; STOLF, N. A. G.; SEFERIAN Jr. P.; PÊGO-FERNANDES, P. M.; PEREIRA-BARRETO, A. C.; PILEGGI, F.; JATENE, A. D. - Limitaçōes da cardiomioplastia no tratamento das cardiomiopatias. Rev. Bras. Cir. Cardiovasc., 7(2):127-135, 1992.

RESUMO: A cardiomioplastia melhora a função ventricular esquerda e a sobrevida de pacientes portadores de cardiomiopatias severas. O objetivo deste estudo é identificar os fatores que influenciaram os resultados da cardiomioplastia em 22 pacientes operados entre maio de 1988 e dezembro de 1991. Todos os pacientes estavam em classe funcional III ou IV, apesar do uso de terapêutica clínica otimizada. Dezoito pacientes tinham diagnóstico de cardiomiopatia idiopática, a cardiomiopatia era chagásica em 2 e isquêmica em 2. Nảo houve óbitos no período pós-operatório imediato e os pacientes foram seguidos por um período médio de 20,5 meses. Nove pacientes faleceram tardiamente e a sobrevida atuarial foi $76,1 \%$ no primeiro ano e $63,8 \%$ no segundo ano de seguimento. Seis pacientes estāo, atualmente, em classe funcional I, e 7 em classe II. A mortalidade e a manutençāo dos sintomas, no primeiro ano pós cardiomioplastia, foi relacionada a ocorrência de tromboembolismo pulmonar e a progressāo da insuficiência cardíaca em pacientes com alteraçōes isquêmicas do enxerto muscular, no pós-operatório imediato (pico de liberaçāo plasmática da creatinoquinase > 1500 U.I.) ( $p=0,03)$. Paralelamente, a elevação da fraçāo de ejeção do ventrículo esquerdo, documentada aos seis meses de seguimento, foi mais importante em pacientes que apresentaram valores menores de liberaçāo da creatinoquinase após a operaçāo $(p=0,02)$. Já o tamanho da cavidade ventricular esquerda pareceu influenciar a variaçāo da fração de ejeção apenas quando foram retirados da análise os pacientes com comprometimento importante do enxerto muscular $(p=0,06)$. Apesar de nāo ter havido influência da classe funcional pré-operatória sobre esse parâmetro e sobre a evoluçăo clínica, no primeiro ano de seguimento, os pacientes operados em classe funcional IV apresentaram uma sobrevida, no segundo ano de pós-operatório, significativamente inferior à dos pacientes operados em classe III $(33,3 \%$ versus $78,1 \%, p=0,04)$. Em conclusão, a melhora da função ventricular esquerda e a melhor evoluçāo clínica após a cardiomioplastia podem ser limitadas pela ocorrência de lesāo isquêmica do enxerto muscular. A condiçāo clínica pré-operatória, bem como o grau de dilataçāo das câmaras ventriculares, são, também, fatores importantes para o sucesso deste procedimento no tratamento das cardiomiopatias.

DESCRITORES: cardiomioplastia; miocardiopatias, cirurgia.

\section{INTRODUÇĀO}

A melhora da função ventricular esquerda e a reversão da insuficiência cardíaca congestiva têm sido documentadas com a utilização da cardiomioplastia, no tratamento das cardiomiopatias terminais 2 2. 3. 14. 18, 24. Paralelamente, foi também observada melhora da sobrevida de pacientes portadores de cardiomiopatias dilatadas com esse procedimento 22,24 .
Por outro lado, resultados controversos a respeito da utilização da cardiomioplastia têm sido relatados ${ }^{8}, 10,11$ sendo, provavelmente, influenciados pela associação dessa operação a outros procedimentos, ou por diferenças nas populaçōes estudadas. Trabalhos recentes têm especulado a influência de fatores, como a classe funcional pré-operatória ${ }^{10}$, o grau de comprometimento da função ventricular ${ }^{14}, 18 \mathrm{e}$ a existência de procedimentos associados ${ }^{3}$ nos resultados da cardiomioplastia.

Trabalho realizado no Instituto do Coração do Hospital das Clínicas da Faculdade de Medicina da Universidade de Săo Paulo. Sáo Paulo, SP. Brasil. Apresentado o $19^{\circ}$ Congresso Nacional de Cirurgia Cardíaca. São Paulo, SP, 7 a 9 de maio, 1992.

- Do Instituto do Coraçáo do Hospital das Clínicas da Faculdade de Medicina da Universidade de São Paulo.

Endereço para separatas: Luiz Felipe P. Moreira. Av. Dr. Enéas Carvalho de Aguiar, 44 - Divisâo Cirúrgica. 05403 São Paulo, SP, Brasil. 
MOREIRA, L. F. P.; BOCCHI, E. A.; STOLF, N. A. G.; SEFERIAN Jr. P.; PÊGO-FERNANDES, P. M.; PEREIRA-BARRETO, A. C.; PILEGGI, F.; JATENE, A. D. - Limitaçōes da cardiomioplastia no tratamento das cardiomiopatias. Rev. Bras. Cir. Cardiovasc., $7(2): 127-135,1992$.

O objetivo deste estudo é identificar fatores que possam limitar os efeitos da cardiomioplastia sobre a função ventricular esquerda, bem como sobre a evolução clínica de pacientes portadores de cardiomiopatias isquêmicas ou dilatadas.

\section{CASUÍSTICA E MÉTODOS}

\section{Populaçāo Estudada}

No período de maio de 1988 a dezembro de 1991, 22 pacientes foram submetidos a cardiomioplastia, no Instituto do Coração. Dezoito pacientes tinham diagnóstico de cardiomiopatia dilatada idiopática, ém 2 a cardiomiopatia era secundária a doença de Chagas e em 2 pacientes era de origem isquêmica. A idade dos pacientes variou de 15 a 57 anos (média: 43,5 anos) e 19 pacientes eram do sexo masculino. Dezesseis pacientes estavam em classe funcional III e 6 em classe IV (New York Heart Association), apesar do uso otimizado de digital, diuréticos e vasodilatadores.

A indicaçāo da cardiomioplastia foi baseada na existência de um comprometimento severo da funçāo ventricular esquerda (fração de ejeçăo de ventrículo esquerdo $<30 \%$ pela angiografia radioisotópica: pressão capilar pulmonar $>15 \mathrm{mmHg}$ ). Foram considerados contra-indicação ao procedimento: o comprometimento importante da funçāo pulmonar, as doenças musculares degenerativas, taquiarritmias não responsivas ao tratamento medicamentoso e a insuficiência importante ou severa da valva mitral. Nos pacientes com cardiomiopatia isquêmica, o cateterismo cardíaco mostrou oclusão total de uma ou de duas artérias coronárias, nāo sendo observada, contudo, existência de angina ou evidências de isquemia pelo estudo da perfusão miocárdica pelo Thallium-201.

\section{Avaliação Não Invasiva da Função Ventricular}

Neste estudo foram analisados dados obtidos pela Doppler-ecocardiografia e pela angiografia radioisotópica no período pré-operatório (Tabela 1) e aos 6 meses de pós-operatório.

A avaliação Doppler-ecocardiográfica foi realizada com um aparelho Aloka SSD-725. As determinaçōes bidimensionais e em modo $M$ foram obtidas com um transdutor de $2,5 \mathrm{MHz}$, sendo utilizado um transdutor de $2 \mathrm{MHz}$ para o Doppler. A análise em modo $\mathrm{M}$ permitiu a medida das dimensōes internas do ventrículo esquerdo e a determinaçăo do encurtamento segmentar daquela câmara. $O$ grau de re- gurgitação através da valva mitral foi quantificado pela ecocardiografia bidimensional e pelo Doppler contínuo e pulsátil.

A angiografia radioisotópica dos ventrículos direito e esquerdo foi obtida após a injeção de hemácias marcadas com Technécio 99m. As imagens cintilográficas foram adquiridas na posição oblíqua anterior esquerda, com uma câmara Ohio Nuclear equipada com um computador Sopha S500 . Os volumes e a fração de ejeção dos ventrículos direito e esquerdo foram calculados por fórmulas padronizadas.

\section{Procedimento Cirúrgico}

A cardiomioplastia foi realizada segundo a técnica descrita por CHACHQUES et alii ${ }^{5}$. 0 músculo grande dorsal esquerdo foi fixado sobre a superfície das câmaras ventriculares, como descrito em publicaçōes anteriores ${ }^{14,25}$, obtendo-se um envolvimento cardio-costal posterior esquerdo ${ }^{\circ}$. Em um dos pacientes, a cardiomioplastia foi associada à realização de um enxerto aorto-coronário de veia safena para a artéria coronária direita, sem a utilização de circulaçăo extracorpórea. Dois eletrodos intramusculares (Medtronic SP 5528) foram implantados no enxerto muscular e um eletrodo epimiocárdico de sensibilidade (Medtronic SP 5548) foi implantado em ventrículo direito ou esquerdo. A estimulação do enxerto muscular foi realizada através de um cardiomioestimulador Medtronic SP 1005, implantado na parede abdominal.

Durante o procedimento cirúrgico e na primeira semana de pós-operatório, foi mantido o uso profilático de drogas inotrópicas positivas e vasodilatadores, com o objetivo de se obterem débito cardíaco elevado e adequada pressāo de perfusão nos órgãos periféricos e no enxerto muscular ${ }^{23}$. Nos primeiros seis dias após a cardiomioplastia, o grau de comprometimento isquêmico do músculo grande

TABELA 1

AVALIAÇĀO DA FUNÇĀO VENTRICULAR NO PRÉ-OPERATÓRIO

\begin{tabular}{|c|c|}
\hline VARIÁVEIS & $M E ́ D I A \pm D P$ \\
\hline $\begin{array}{l}\text { Raio X de Tórax: } \\
\text { Índice Cardiotorácico (\%) }\end{array}$ & $58,6 \pm 3,2$ \\
\hline $\begin{array}{l}\text { Doppler-ecocardiografia: } \\
\text { Diâmetro Diastólico de V E (mm) } \\
\text { Encurtamento Segmentar VE (\%) }\end{array}$ & $\begin{array}{l}73,6 \pm 7,6 \\
11,6 \pm 2,4\end{array}$ \\
\hline $\begin{array}{l}\text { Angiografia Radioisotópica: } \\
\text { Fraçāo de Ejeção de V D (\%) } \\
\text { Fração de Ejeção de V E (\%) }\end{array}$ & $\begin{array}{l}23,2 \pm 5,4 \\
20,4 \pm 3,6\end{array}$ \\
\hline
\end{tabular}

$V E=$ Ventrículo esquerdo; $V D=$ Ventrículo direito. 
MOREIRA, L. F. P.; BOCCHI, E. A.; STOLF, N. A. G.; SEFERIAN Jr. P.; PÊGO-FERNANDES, P. M.; PEREIRA-BARRETO, A. C.; PILEGGI, F.; JATENE, A. D. - Limitaçōes da cardiomioplastia no tratamento das cardiomiopatias. Rev. Bras. Cir. Cardiovasc., $7(2): 127-135,1992$.

dorsal esquerdo foi avaliado pela dosagem, a cada 12 horas, dos níveis plasmáticos da creatinoquinase.

A estimulação elétrica do enxerto muscular foi iniciada duas semanas após a operaçāo, sendo seguido o protocolo de estimulação muscular progressiva proposto por CARPENTIER \& CHACHQUES ${ }^{4}$ e CHACHQUES et alii ${ }^{6}$. Após o período de condicionamento muscular, os parâmetros de estimulaçāo do músculo grande dorsal foram ajustados, com o objetivo de se manter uma contração efetiva do enxerto, sincronizada a todos os batimentos cardíacos ${ }^{14,25}$.

\section{Fatores Analisados no Seguimento Pós-Operatório}

A evolução clínica, no primeiro ano pós cardiomioplastia, foi avaliada nos pacientes que completaram esse período, sendo considerados como casos de sucesso cirúrgico apenas os pacientes que se mantiveram em classe funcional I ou II, após os seis meses de pós-operatório. A sobrevida tardia dos pacientes foi também analisada e os pacientes que evoluíram para óbito foram submetidos, sempre que possível, à necrópsia. Neste estudo, foram avaliadas as características macro e microscópias do enxerto muscular.

Foi estudada, a seguir, a correlação entre a evoluçāo clínica e entre a variaçāo de fração de ejeçāo do ventrículo esquerdo e os seguintes fatores: classe funcional, dimensāo da cavidade ventricular esquerda, fraçāo de ejeção dos ventrículos direito e esquerdo e grau de insuficiência mitral no período pré-operatório, grau de recobrimento dos ventrículos pelo enxerto e pico plasmático da creatinoquinase no pós-operatório imediato.

\section{GRÁFICO 1}

CURVA DE VARIAÇĀO DOS NÍVEIS PLASMÁTICOS DA CREATINOQUINASE NO PÓS-OPERATÓRIO IMEDIATO DA CARDIOMIOPLASTIA (MEDIA $\pm D P)$

\section{VARIaço da creatimokimase}

U.1.

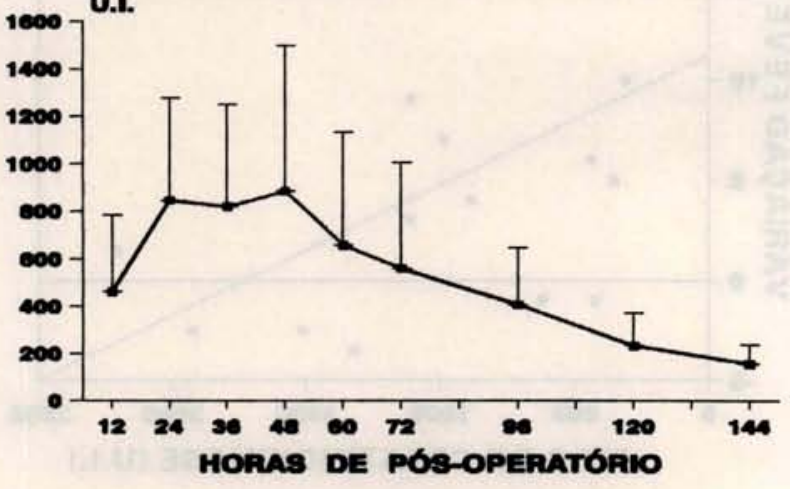

\section{Análise Estatística}

Os dados obtidos no período pré-operatório e no seguimento pós-operatório foram comparados através da análise de variância de medidas repetidas ${ }^{16}$. A relação entre os parâmetros analisados e a evolução clínica dos pacientes no primeiro ano de seguimento pós cardiomioplastia foi avaliada pelo teste de Qui quadrado ${ }^{16}$. A influência dos mesmos parâmetros sobre a variaçăo da fraçāo de ejeçāo do ventrículo esquerdo foi testada por meio de regressāo linear ${ }^{16}$. As curvas de sobrevida foram obtidas pelo método atuarial e a diferença entre as curvas foi analisada pelo método de Log-Rank ${ }^{16}$. Valores de $p<0,05$ foram considerados significantes. Os resultados são apresentados nos valores médios \pm 1 desvio padrāo.

\section{RESULTADOS}

\section{Seguimento Pós-Operatório}

Não houve óbitos no período pós-operatório imediato. Dois pacientes apresentaram baixo débito cardíaco transitório, seguido de insuficiência de múltiplos órgãos. O Gráfico 1 mostra a curva dos valores médios de liberaçāo plasmática da creatinoquinase nos seis primeiros dias de pós-operatório. Os valores observados confirmam a existência de comprometimento esquêmico do enxerto muscular em todos os pacientes. Em 4 pacientes, o pico de liberaçāo da creatinoquinase foi maior do que 1400 U.I., sendo que 2 desses casos eram os pacientes que haviam apresentado baixo débito transitório e comprometimento de múltiplos órgãos.

O seguimento pós-operatório variou de dois a 48 meses, sendo em média de 20,5 $\pm 13,7$ meses. Dos 19 pacientes com pelo menos um ano de seguimento, 5 foram a óbito no primeiro ano pós cardiomioplastia. As causas foram: tromboembolismo pulmonar seguido de infecçāo pulmonar ou choque cardiogênico em 2 pacientes e progressāo da insuficiência cardíaca associada a processos infecciosos ou a acidente vascular cerebral em 3 . Outros 3 pacientes apresentaram períodos de descompensaçāo cardíaca importante após os seis meses de pós-operatório, sendo que 2 permaneceram em classe funcional III. Seis pacientes estavam em classe funcional I e $6 \mathrm{em}$ classe II, ao final de um ano de seguimento.

No seguimento de dois e três anos de pósoperatório, outros 4 pacientes aforam a óbito, sendo observada uma sobrevida atuarial de $76,1 \pm 9 \%$ no primeiro ano, de $63,8 \pm 11,6 \%$ no segundo ano e de $48,6 \pm 13,2 \%$ no terceiro ano após a cardio- 
MOREIRA, L. F. P.; BOCCHI, E. A.; STOLF, N. A. G.; SEFERIAN Jr. P.; PÊGO-FERNANDES, P. M.; PEREIRA-BARRETO, A. C.; PILEGGI, F.; JATENE, A. D. - Limitaçōes da cardiomioplastia no tratamento das cardiomiopatias. Rev. Bras. Cir. Cardiovasc., $7(2): 127-135,1992$.

mioplastia. As causas de óbito nesses casos foram: progressāo da insuficiência cardíaca em 2 pacientes e morte súbita em 2. Os pacientes sobreviventes mantiveram-se em classe funcionall ou II, nāo tendo apresentado novos episódios de descompensaçăo.

O estudo radioisotópico demonstrou que a fração de ejeçāo do ventrículo esquerdo se elevou de $20,7 \pm 4,2 \%$ para $25,7 \pm 7,1 \%$ aos seis meses de pós-operatório $(p=0,01)$, sendo esse parâmetro reflexo dos efeitos hemodinâmicos da cardiomioplastia já analisados em publicaçōes anteriores ${ }^{2,14,23-25}$.

\section{Fatores Prognósticos}

O estudo da correlação entre as características pré e pós-operatórias analisadas neste trabalho e a evolução clínica dos pacientes no primeiro ano de seguimento após a cardiomioplastia mostraram que os pacientes que apresentaram niveis de creatinoquinase maiores do que 1400 U.I. evoluíram para óbito ou com manutenção dos sintomas de descompensaçāo cardíaca, naquele período $\left(X^{2}=4,28\right.$; $p=0,03$ ). $O$ Gráfico 2 confirma, através de regressāo linear, a relação significativa entre os níveis de creatinoquinase e a condição clínica dos pacientes, ao final de um ano de pós-operatório. Paralelamente, nos pacientes que foram a óbito e que tinham níveis elevados de creatinoquinase após a cardiomioplastia, foi constatado, na necrópsia, que os enxertos musculares apresentavam sinais de fibrose e atrofia importante.

\section{GRÁFICO 2}

CORRELAÇĀO ENTRE O PICO DA CREATINOQUINASE NO PÓS-OPERATÓRIO IMEDIATO E A EVOLUÇÄO CLÍNICA AO FINAL DO 1ANO DE SEGUIMENTO PÓS CARDIOMIOPLASTIA.

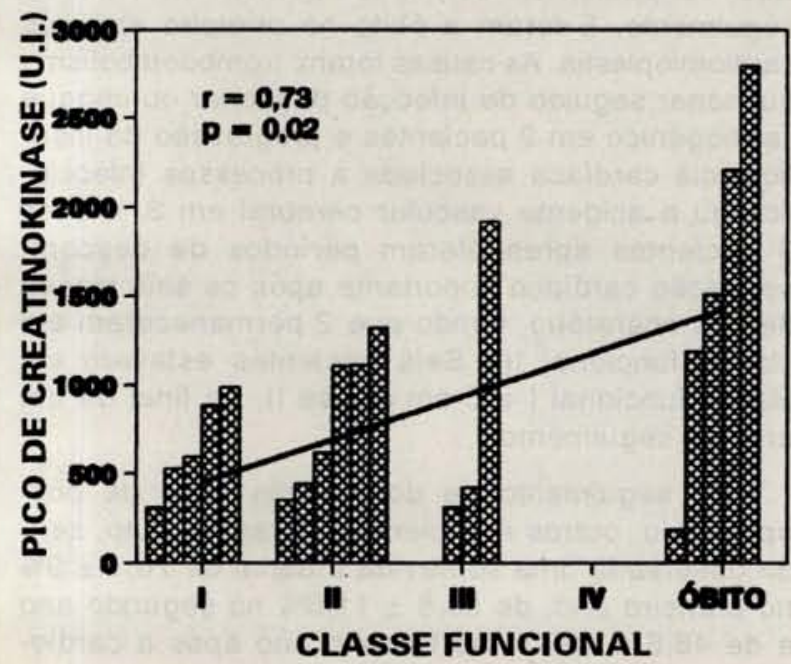

Nāo foi observada qualquer relação entre os outros parâmetros estudados e a evoluçāo clínica dos pacientes no primeiro ano após cardiomioplastia. Por outro lado, a análise da curva de sobrevida até três anos de seguimento demonstrou que os pacientes operados em classe funcional IV tiveram mortalidade maior do que os operados em classe III (Gráfico 3).

A variação da fraçāo de ejeção do ventrículo esquerdo após a cardiomioplastia também foi significativamente influenciada pelos níveis de crea-

\section{GRÁFICO 3}

CURVA ATUARIAL DE SOBREVIDA DOS PACIENTES SUBMETIDOS A CARDIOMIOPLASTIA CONFORME A CLASSE FUNCIONAL (C.F.) PRE-OPERATÓRIA. (NUMMEROS) INDICAM OS PACIENTES SOB RISCO NO PERIODO

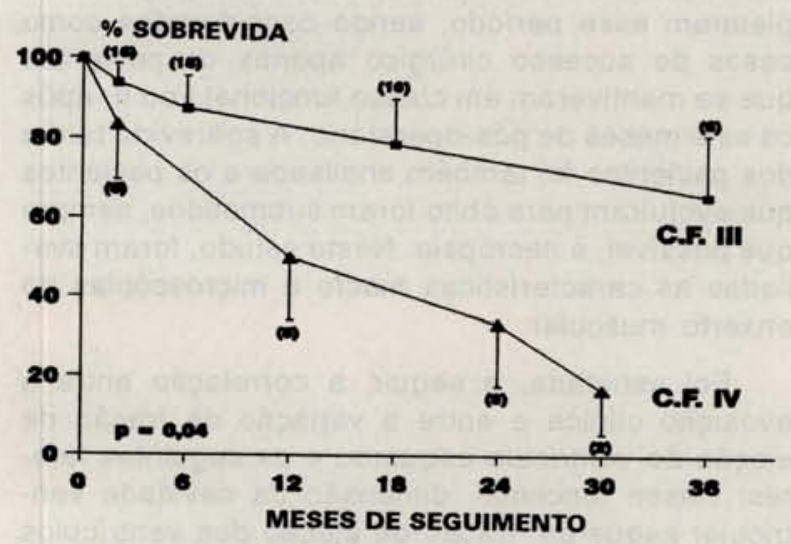

GRÁFICO 4

CORRELAÇĀO ENTRE O PICO PLÁSMÁTICO DA CREATINOQUINASE NO PÓS-OPERATÓRIO IMEDIATO E A VARIAÇĀO DA FRAÇĀO DE EJEÇĀO DO VENTRICULO ESQUERDO (FEVE) APÓS A CARDIOMIOPLASTIA

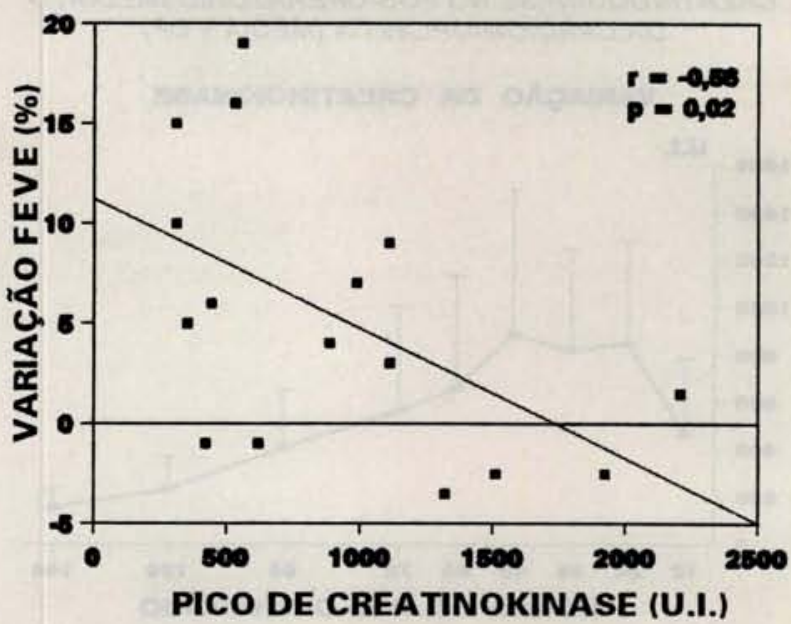


MOREIRA, L. F. P.; BOCCHI, E. A.; STOLF, N. A. G.; SEFERIAN Jr. P.; PÊGO-FERNANDES, P. M.; PEREIRA-BARRETO, A. C.; PILEGGI, F.; JATENE, A. D. - Limitaçōes da cardiomioplastia no tratamento das cardiomiopatias. Rev. Bras. Cir. Cardiovasc., 7(2):127-135, 1992.

tinoquinase, no pós-operatório imediato, como mostra - Gráfico 4. Já o tamanho da cavidade ventricular esquerda parece ter influenciado a variaçăo da fração de ejeção apenas quando foram retirados da análise os pacientes com grave comprometimento isquêmico do enxerto muscular (Gráfico 5). Não houve qualquer relação entre o grau de recobrimento das câmaras ventriculares pelo enxerto e a melhora da fração de ejeção após a cardiomioplastia.

\section{GRÁFICO 5}

CORRELAÇĀO ENTRE O DIÂMETRO DIASTÓLICO DO VENTRICULO ESQUERDO (VE) NO PRÉ-OPERATÓRIO E A VARIAÇĀO DA FRAÇĀO DE EJEÇĀO DO VE (FEVE) APÓS A CARDIOMIOPLASTIA

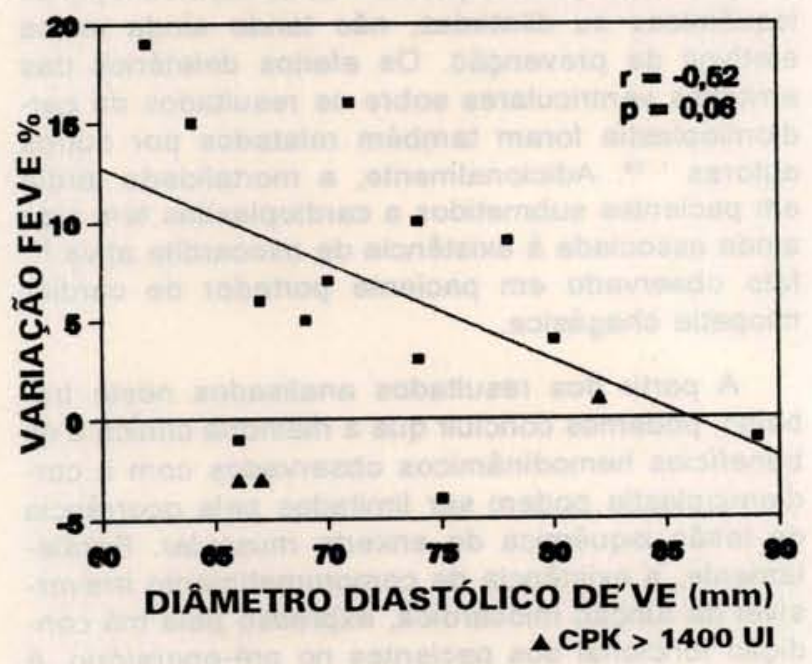

\section{COMENTÁRIOS}

Apesar dos resultados demonstrados em estudos experimentais ${ }^{15}$, 17, 21, a experiência clínica com a utilizaçāo da cardiomioplastia no tratamento das cardiomiopatias terminais tem-se mostrado controversa e com resultados limitados a longo prazo. A melhora da funçāo ventricular esquerda ${ }^{2,13,14,24,25}$, assim como a melhora da qualidade de vida ${ }^{19,25} \mathrm{e}$ o aumento da sobrevida ${ }^{22,24}$ têm sido relatados, principalmente quando esse procedimento é empregado no tratamento de pacientes portadores de cardiomiopatias dilatadas. Por outro lado, experiências de caráter multicêntrico ${ }^{10} \mathrm{e}$ casuístidas que incluem casos com procedimentos associados ${ }^{8,11 .}$ ${ }^{18}$ têm apresentado resultados pouco conclusivos. Além disso, alguns trabalhos têm mostrado mortalidade tardia importante com a cardiomioplastia 10 . ${ }^{18} \mathrm{e}$ não existem ainda estudos bem conduzidos sobre os fatores que podem influenciar os resultados imediatos e tardios desse procedimento.

Neste trabalho, foi demonstrado que o grau de comprometimento funcional dos pacientes no préoperatório e a existência de comprometimento isquêmico importante do enxerto muscular durante a cardiomioplastia podem limitar a melhora da função ventricular e a evolução clínica dos pacientes no pós-operatório.

Do ponto de vista teórico, o grau de eficiência da cardiomioplastia seria maior se fosse possível se envolver totalmente a superfície das câmaras ventriculares com um enxerto muscular íntegro e de grande tamanho e espessura. Devido a suas características anatômicas, o músculo grande dorsal tem sido o enxerto escolhido para esse procedimento 5 . A perfusão dominante do grande dorsal é obtida a partir do ramo anterior da artéria tóraco-dorsal, fato que, associado à existência de um pedículo nervoso único, permite a sua rotação para o interior da cavidade torácica. Por outro lado, têm sido descritas anastomoses com vasos colaterais na face lateral do grande dorsal ${ }^{29} \mathrm{e}$ a existência de vasos perfurantes a partir da parede torácica ${ }^{12}$. Em conseqüência, apesar da preservação do pedículo vásculo-nervoso principal durante a cardiomioplastia, a queda da perfusão muscular pode ocorrer principalmente na região distal do enxerto. Esse fato tem sido descrito em estudos experimentais, que mostram também que a normalizaçāo da perfusão do enxerto no pós-operatório pode demorar de duas a três semanas ${ }^{20}$. Outros mecanismos podem ainda influir na perfusão do enxerto muscular após a cardiomioplastia, como a ocorrência de baixo débito cardíaco e de episódios de hipotensão no pós-operatório imediato, levando a isquemia e a lesão muscular irreversível.

A creatinoquinase, por ser uma enzima que existe principalmente nos músculos esqueléticos, tem sido utilizada como índice estimativo do grau de lesão desses músculos ${ }^{7}$. A liberação dessa enzima é, provavelmente, resultado da quebra isquêmicas irreversíveis ${ }^{27}$. Neste trabalho, níveis séricos elevados de creatinoquinase foram observados na primeira semana após a cardiomioplastia, em todos os pacientes, sendo documentada uma nítida correlaçāo entre o grau de comprometimento do enxerto muscular medido pela creatinoquinase e os resultados clínicos e laboratoriais desse procedimento, durante o primeiro ano de seguimento. Em 4 casos, a lesāo do enxerto muscular foi mais severa, não sendo observado qualquer benefício após a cardiomioplastia, provavelmente pela existência de atrofia do grande dorsal, conforme documentado em estudo anátomo-patológico.

Em relação à influência do grau de comprometimento funcional dos pacientes no pré-operatório sobre os resultados da cardiomioplastia, a indicaçāo desse procedimento em pacientes em classe funcional IV tem sido relacionada a mortalidade 
MOREIRA, L. F. P.; BOCCHI, E. A.; STOLF, N. A. G.; SEFERIAN Jr. P.; PÊGO-FERNANDES, P. M.; PEREIRA-BARRETO, A. C.; PILEGGI, F.; JATENE, A. D. - Limitaçōes da cardiomioplastia no tratamento das cardiomiopatias. Rev. Bras. Cir. Cardiovasc., 7(2):127-135, 1992.

imediata elevada, em alguns Centros ${ }^{3,10}$. Neste estudo, embora nāo tenham ocorrido óbitos hospitalares, os pacientes operados em classe IV apresentaram, por outro lado, mortalidade tardia importante. As causas nāo tiveram, portanto, relaçāo direta com a cardiomioplastia e resultaram, provavelmente, da progressão da cardiomiopatia de base. Esse fato, que ocorreu precocemente, apesar dos benefícios hemodinâmicos documentados com esse procedimento, deve estar relacionado à existência de um grau irreversível de comprometimento da função miocárdica. A esse respeito, a falta de resposta de pacientes portadores de cardiomiopatias severas à terapêutica medicamentosa tem sido associada à existência de uma baixa reserva funcional do miocárdio ${ }^{30}$

Outros índices pré-operatórios do grau de comprometimento da funçāo ventricular nāo tiveram relação com os resultados da cardiomioplastia, neste estudo, provavelmente por causa da gravidade dos pacientes analisados. Apenas o grau de dilataçāo da cavidade ventricular esquerda parece ter influenciado a variaçāo da fraçāo de ejeçāo do ventrículo esquerdo no pós-operatório. Esse fato, já sugerido em publicaçōes anteriores ${ }^{14}{ }^{25}$, foi importante apenas quando foram retirados da análise os pacientes com alteraçōes isquêmicas do enxerto muscular, devendo ser reavaliado futuramente, com maior número de casos. Outros estudos têm relacionado a ocorrência de resultados desfavoráveis com a cardiomioplastia à existência de comprometimento biventricular ${ }^{3,18}$. Esses dados foram observados, no entanto, em pacientes portadores de cardiomiopatia isquêmica, uma indicaçāo que difere da maioria dos pacientes desse estudo, que incluíu, predominantemente, casos de cardiomiopatia dilatada idiopática.

A influência de aspectos da técnica cirúrgica utilizada sobre os resultados da cardiomioplastia tem sido também investigada. A esse respeito, o tipo de orientação das fibras do enxerto muscular ${ }^{15}$ e o grau de recobrimento das câmaras ventriculares pelo grande dorsal ${ }^{21}$ são fatores ainda controversos. Já a realização de procedimentos asso- ciados, que necessitem a utilização de circulaçăo extracorpórea, parece ser determinante de um risco hospitalar elevado ${ }^{3}$, nāo havendo, no entanto, estudos sobre a sua influência na evoluçāo tardia da cardiomioplastia.

Finalmente, é importante lembrar que as causas de óbito no pós-operatório tardio da cardiomioplastia nem sempre estão relacionadas ao procedimento realizado. O trombo-embolismo pulmonar ocorreu em 2 apacientes nessa série e 2 pacientes, que tinham arritmias ventriculares importantes no pós-operatório, morreram subitamente. Essas complicaçōes năo sāo, provavelmente, influenciadas pela cardiomioplastia e ocorrem com freqüência em pacientes portadores de cardiomiopatias isquêmicas ou dilatadas, nāo tendo ainda meios efetivos de prevenção. Os efeitos deletérios das arritmias ventriculares sobre os resultados da cardiomioplastia foram também relatados por outros autores 1, 26. Adicionalmente, a mortalidade tardia em pacientes submetidos a cardioplastias tem sido ainda associada à existência de miocardite ativa ${ }^{28}$, fato observado em paciente portador de cardiomiopatia chagásica.

A partir dos resultados analisados neste trabalho, podemos concluir que a melhoria clínica es benefícios hemodinâmicos observados com a cardiomioplastia podem ser limitados pela ocorrência de lesāo isquêmica do enxerto muscular. Paralelamente, a existência de comprometimento irreversível da funçāo miocárdica, expresso pela má condição funcional dos pacientes no pré-operatório, é fator que restringe os resultados da cardiomioplastia a longo prazo. A severidade da dilataçāo das câmaras ventriculares pode limitar a atuaçāo da cardiomioplastia sobre a funçăo ventricular, năo representado, contudo, fator de mau prognóstico tardio.

Este trabalho abre novas perspectivas no estudo da cardiomioplastia, sugerindo a pesquisa de mecanismos de preservaçăo da integridade do enxerto muscular e a restriçāo do emprego desta técnica a pacientes com comprometimento menos severo da função ventricular. 
MOREIRA, L. F. P.; BOCCHI, E. A.; STOLF, N. A. G.; SEFERIAN Jr. P.; PÊGO-FERNANDES, P. M.; PEREIRA-BARRETO, A. C.; PILEGGI, F.; JATENE, A. D. - Limitaçōes da cardiomioplastia no tratamento das cardiomiopatias. Rev. Bras. Cir. Cardiovasc., $7(2): 127-135,1992$.

RBCCV 44205-171

MOREIRA, L. F. P.; BOCCHI, E. A.; STOLF, N. A. G.; SEFERIAN Jr., P.; PÊGO - FERNANDES, P. M.; PEREIRA-BARRETO, A. C.; PILEGGI, F.; JATENE, A.D. - Cardiomyoplasty limitations in the treatment of severe cardiomyopathies. Rev. Bras. Cir. Cardiovasc., 7(2):127-135,1992.

ABSTRACT: Dynamic cardiomyoplasty improves left ventricular function and survival of patients with severe cardiomyopathies. The purpose of this study was to investigate the factors influencing cardiomyoplasty results in 22 patients operated upon at the Heart Institute. All patients were in New York Heart Association class III or IV, despite the use of maximal medical therapy. Eighteen patients had idiopathic dilated cardiomyopathy, in two patients the cardiomyopathy was due to Chagas' disease and in two due to ischemic ethiology. There were no operative death and patients were followed up for a mean of 20.5 months. Nine patients died at late follow-up period, and actuarial survival rates were $76.1 \%$ at 1 year and $63.8 \%$ at 2 years of follow-up. Six patients are presently in functional class I and six in class II. The mortality and the absence of functional improvement at 1 year were associated to episodes of pulmonary thromboelbolysm and to heart failure progression in patients with severe muscle flap ischemic compromise at the immediate postoperative period (creatinokinase peak level > 1400 l.U.) $(p=0.03)$. In addition, the improvement of left ventricular ejection fraction at 6 months of follow-up was more significant in patients who presented lower values of creatinokinase after the surgical procedure $(p=0.02)$. Otherwise, the influence of left ventricular dimension on ejection fraction changes was documented only when patients with severe muscle flap compromise were withdrawn from the analysis $(p=0.06)$. Despite the absence of functional class influence on 1 year results of cardiomyoplasty, patients operated upon in class IV presented a less significant survival than patients operated upon in class III at 2 years of follow-up $(33.3 \%$ versus $78.1 \%, p=0.04)$. In conclusion, quality of life and left ventricular function improvement after cardiomyoplasty may be limited by muscle flap ischemic compromise. Patient's condition prior to surgery and the degree of left ventricular dilation may also influence cardiomyoplasty results in patients with severe cardiomyopathies.

DESCRIPTORS: cardiomyoplasty; cardiomyopathies, surgery.

\section{REFERÊNCIAS BIBLIOGRÁFICAS}

1 ALMADA, H.; MOLTENI, L.; FERREIRA, R.; ORTEGA, D. - Clinical experience with dynamic cardiomyoplasty. J. Cardiac Surg.; 5: 193-198, 1990.

2 BOCCHI, E. A.; BELLOTTI, G.; MOREIRA, L. F. P.; MORAES, A. V.; GAMA, M.; BARRETTO, A. C. P.; STOLF, N. A. G.; JATENE, A. D. - Regional wall motion, ejection fraction and geometry of left ventricle after cardiomyoplasty. Circulation, 84(Supl. 2) 355 , 1991.

3 CARPENTIER, A. \& CHACHQues, J. C. - Clinical dynamic cardiomyoplasty: method and outcome. Sem. Thorac. Cardiovasc. Surg., 3: 136-139, 1991.

4 CARPENTIER, A. \& CHACHQUES, J. C. - Latissimus dorsi cardiomyoplasty to increase cardiac output. In: RÁBAGO, G. \& COOLEY, D. A. (eds.) Heart valve replacement: current status and future trends. Mount Kisco, Futura, 1987. p.473-486.

5 CHACHQUES, J. C.; GRANDJEAN, P. A.; CARPENTIER, A. - Latissimus dorsi dynamic cardiomyoplasty. Ann. Thorac. Surg., 47: 600-604, 1989.

6 CHACHQUES, J. C.; GRANDJEAN, P. A.; SCHWARTZ, K.; MIHAILEANU, S.; FARDEAU, M.;
SWYNGHEDAUW, B.; FONTALIRAN, F.; ROMERO, N.; WISNEWSKY, C.; PERIER, P.; CHAUVAUD, S.; BOURGEOIS, I.; CARPENTIER, A. - Effect of latissimus dorsi dynamic cardiomyoplasty on ventricular function. Circulation, 78 (Supl. 3): 203 216, 1988.

7 CHIU, D.; WANG, H. H.; BLUMENTHAL, M. R. - Creatino phosphokinase release as a measure of the tourniquet effect on skeletal muscle. Arch. Surg., 111: $71-74,1976$.

8 DELAHAYE, F.; JEGADEN, O.; MONTAGNA, P.; DESSEIGNE, P.; BLANC, P.; VENDRINNE, C.; TOUBOUL, P.; SAINT-PIERRE, A.; PERINETTI, M.; ROSSI, R.; ITTI, R.; MIKAELOFF, P. - Latissimus dorsi cardiomyoplasty in severe congestive heart failure: the Lyon experience. J. Cardiac Surg., 6(Supl.): 106-112, 1991.

9 FURNARY, A. P.; CHRISTLIEB, I. Y.; MAGOVERN, J. A.; KAO, R. L.; MAGOVERN, G. J. - Wrap nomenclature for latissimus dorsi cardiomyoplasty. Sem. Thorac. Cardiovasc. Surg., 3: 132-135, 1991.

GRANDJEAN, P. A.; AUSTIN, L.; CHAN, S.; TERPSTRA, B.; BOURGEOIS, I. - Dynamic cardiomyoplasty: clinical follow-up results. J. Cardiac. Surg., 6(Supl.): 80-88, 1991.

11 HAGEgE, A. A.; DESNOS, M.; CHACHQUES, J. C.; 
MOREIRA, L. F. P.; BOCCHI, E. A.; STOLF, N. A. G.; SEFERIAN Jr. P.; PÊGO-FERNANDES, P. M.; PEREIRA-BARRETO, A. C.; PILEGGI, F.; JATENE, A. D. - Limitaçōes da cardiomioplastia no tratamento das cardiomiopatias. Rev. Bras. Cir. Cardiovasc., $7(2): 127-135,1992$.

CARPENTIER, A. - Follow-up after dynamic cardiomyoplasty: preliminary report. Lancet, 335: 1122-1124, 1990.

HECKLER, F. R. \& WHITE, M. J. - Isolation of skeletal muscle for biomechanical circulatory assist. Sem. Thorac. Cardiovasc. Surg., 3: 128-131, 1991.

HILL, A. B. \& CHIU, R. C. - Dynamic cardiomyoplasty for treatment of heart failure. Clin. Cardiol., 12: $681-688,1989$.

JATENE, A. D.; MOREIRA, L. F. P.; STOLF, N. A. G.; BOCCHI, E. A.; SEFERIAN Jr., P.; FERNANDES, P. M. P.; ABENSUR, $H$. - Left ventricular function changes after cardiomyoplasty in patients with dilated cardiomyopathy. J. Thorac. Cardiovasc. Surg., 102: 132-139, 1991.

KAO, R. L.; CHRISTLIEB, I. Y.; MAGOVERN, G. J.; PARK, S. B.; MAGOVERN. Jr., G. J. - The importance of skeletal muscle fiber orientation for dynamic cardiomyoplasty. J. Thorac. Cardiovasc. Surg., 99: $134-140,1990$.

KRAMER, M. S. - Clinical epidemiology and biostatistics: a primer for clinical investigations and decision-makers. Berlin, Springer-Verlag. 1988, $286 \mathrm{p}$.

17 LEE, K. F.; DIGNAN, R. J.; PARMAR, J. M.; DYKE, C. M.; BENTON, G.; YEH Jr., T.; ABD-ELFATTAH, A. S.; WECHSLER, A. S. - Effects of dynamic cardiomyoplasty on left ventricular perfomance and myocardial mechanics in dilated cardiomyopathy. J. Thorac. Cardiovasc. Surg., 102: 124-131, 1991.

MAGOVERN, J. A.; FURNARY, A. P.; CHRISTLIEB, I. Y.; KAO, R. L.; PARK, S. B.; MAGOVERN, G. J. Indications and risk analysis for clinical cardiomyoplasty. Sem. Thorac. Cardiovasc. Surg., 3: 145-148, 1991.

MAIO, S. A. B.; LAMOSA, B. W. R.; BOCCHI, E. A.; MOREIRA, L. F. P.; STOLF, N. A. G.; BARRETO, A. C. P.; BELLOTTI, G.; PILLEGGI, F.; JATENE, A. D. - Qualidade de vida após cardiomioplastia. Rev. Soc. Cardiol. Est. São Paulo, 1 (Supl. 2): 7 $12,1991$.

MANNION, J. D.; VELCHIK, M. A.; ACKER, M. A.; HAMMOND, R.; ALAVA, A.; STEPHENSON, L. W. - Transmural blood flow of multilayered latissimus dorsi skeletal muscle ventricles during circulatory assistance. Trans. Am. Soc. Artif. Intern. Organs, 32:454-460, 1986.

MOREIRA, L. F. P.; CHAGAS, A. C. P.; CAMARANO, G. P.; LEIRNER, A.; PÊGO-FERNANDES, P. M.; LUZ, P. L.; STOLF, N. A. G.; JATENE, A. D. Cardiomyoplasty benefits in experimental myocardial dysfuntion. J. Cardiac Surg., 4: 164-170. 1989. PÊGO-FERNANDES, P. M.; STOLF, N. A. G.; PE-
REIRA-BARRETTO, A. C.; JATENE, A. D. - Survival improvement with dynamic cardiomyoplasty in patients with dilated cardiomyopathy. Circulation, 84 (Supl. 3): 296-302, 1991.

23 MOREIRA, L. F. P.; STOLF, N. A. G.; BOCCHI , E. A.; AULER Jr., J. O. C.; PÊGO-FERNANDES, P. M.; MORAES, A. V.; MENEGHETTI, J. C.; PEREIRABARRETTO, A. C.; PILEGGI, F.; JATENE, A. D. Perspectivas da cardiomioplastia no tratamento das cardiomiopatias. Rev. Bras. Cir. Cardiovasc., 4: 21-31, 1989.

MOREIRA, L. F. P.; STOLF, N. A. G.; BOCCHI, E. A.; SEFERIAN Jr., P.; PÊGO-FERNANDES, P. M.; PEREIRA-BARRETTO, A. C.; ABENSUR, H.; MENEGHETTI, J. C.; JATENE, A. D. - Resultados da cardiomioplastia no tratamento da cardiomiopatia dilatada. Rev. Bras. Cir. Cardiovasc., 6: 85-95, 1991.

25 MOREIRA, L. F. P.; STOLF, N. A. G.; JATENE, A. D. - Benefits of cardiomyoplasty for dilated cardiomyopathy. Sem. Thorac. Cardiovasc. Surg., 3: $140-144,1991$.

ODIM, J. N. K.; BURGESS, J. H.; WILLIAMS, B. H.; BLUNDELL, P. E.; RABINOVITCH, M. A.; STEWART, J. A.; LOUGH, J. O.; CHIU, R. C. - Pathophysiology of dynamic cardiomyoplasty: a clinicopathological case study. J. Cardiac Surg., 5: 336$346,1990$.

27 PULEO, P. \& ROBERTS, R. - Early biochemical markers of myocardial necrosis. In: PEPINE, C. J. (ed.) Acute myocardial infarction. Philadelphia, F. A. Davis, 1989. p. 143-154. (Cardiovascular Clinics).

28 ROSSI, M.; BRAILE, D. M.; SOUZA, D. R. S.; SANTOS, J. L. V.; THEVENARD, R. S.; PINTO, G. H.; SUZIGAN, S.; GRECCO, O. - Dynamic cardiomyoplasty in chronic Chagas' heart disease: clinicopathological data. Ann. Thorac. Surg., 51: 649-651, 1991.

29 SOLA, O. M.; KAKULAS, B. A.; HAINES, L. C.; THOMAS, R.; SHOJI, Y.; FUJIMURA, Y.; HAYASHIDA, N.; SAUVAGE, L. R. - Morphology and histology of the latissimus dorsi muscle. Sem. Thorac. Cardiovasc. Surg., 3: 124-127, 1991.

30 STEVENSON, L. W.; TILLISCH, J. H.; HAMILTON, M. - Importance of hemodynamic response to therapy in predicting survival with ejection fraction $<20 \%$ secondary to ischemic or nonischemic dilated cardiomyopathy. Am. J. Cardiol., 66: 1348-1354, 1990.

\section{Discussão}

DR. ROBERTO V. ARDITO São José do Rio Preto, SP

No Instituto de Moléstias Cardiovasculares de São José do Rio Preto - SP (IMC), no intervalo de 
MOREIRA, L. F. P.; BOCCHI, E. A.; STOLF, N. A. G.; SEFERIAN Jr. P.; PÊGO-FERNANDES, P. M.; PEREIRA-BARRETO, A. C.; PILEGGI, F.; JATENE, A. D. - Limitaçōes da cardiomioplastia no tratamento das cardiomiopatias. Rev. Bras. Cir. Cardiovasc.,
7(2):127-135, 1992.

junho de 88 a março de 92 operamos 15 pacientes, sendo 12 do sexo masculino e 3 do sexo feminino, com idade média de 39,6 anos, variação de 22 a 58 anos. A indicação foi miocardiopatia dilatada idiopática em 9 , sendo 1 paciente com cardiomiopatia periparto e 6 com cardiopatia chagásica crônica. Os cardio-estimuladores foram: 12 MED.SP/1005, 1 Diplos 06 e 7 Myos BP. Tivemos 5 óbitos que ocorreram nos pacientes chagásicos: 3 por ICC, 1 por taquicardia ventricular, 1 por embolia pulmonar no pós-operatório. Dos pacientes com ICC, 1 teve reagudização da doença de Chagas, indo a óbito 4 meses após cirurgia. A fração de ejeção média dos pacientes no pré-operatório foi de $34,2 \%$, com uma variação de $22 \%$ a $49 \%$ e no pós-operatório uma fração de ejeção média de $49,7 \%$, com uma variação de $31 \%$ a $77,7 \%$ demonstrando um aumento médio de $15,7 \%$ da fração de ejeção do VE. A fração de ejeção nos pacientes com cardiopatia chagásica teve melhora discreta de 2,8 pontos percentuais, enquanto para os demais, de 18,8 pontos percentuais em média. Nós concordamos com - Dr. Felipe: quando se faz a correlaçāo da evolução clínica e a $\mathrm{FE}$, os pacientes com classe funcional IV da NYHA apresentam mortalidade maior, o que nos leva a pensar se há benefício da cirurgia nesses pacientes. Outros autores, como o Dr. Timothy Hooper, vêm pondo em dúvida esse procedimento. Em recente editorial publicado no British Heart Journal (outubro/91), ele diz que fica difícil demonstrar uma melhora hemodinâmica nesses pacientes, uma vez que, pela própria lei de Laplace, năo existia vantagem mecânica evidente, além do que o músculo esquelético, treinado para ser resistente à fadiga, reduz sua força contrátil e diminui sua velocidade de contração, mas, de qualquer forma, refere o próprio Dr. Timothy, que o músculo grande dorsal colocado em volta do coraçāo reduziria a tensāo da parede, em virtude de um aumento efetivo da espessura dos ventrículos envolvidos. Somos da opiniāo que os pacientes com miocardiopatia dilatada que ainda apresentam certo grau de reserva miocárdica e se encontram entre classe funcional III e IV se beneficiam muito com a cirurgia. Mesmo que nāo observemos melhora efetiva na sua
$\mathrm{FE}$, contém o avanço da doença e melhora sobremaneira a sintomatologia dos pacientes, elevandoos à classe funcional II e I, como o próprio $\mathrm{Dr}$. Felipe vem demonstrando. Muito obrigado.

\section{DR. MOREIRA \\ (Encerrando)}

Gostaria de agradecer ao Dr. Roberto Ardito pelos comentários realizados. A experiência do grupo de São José do Rio Preto nos trouxe, sem dúvida, informaçōes importantes a respeito dos problemas observados com a indicação da cardiomioplastia no tratamento da miocardiopatia secundária à cardiopatia chagásica. Como demonstrado pelos dados apresentados, os pacientes portadores de doença de Chagas, apesar de melhorarem após o procedimento cirúrgico, podem vir a falecer em decorrência de fatores relativos à doença de base, como a presença de arritmias severas ou de miocardite ativa. Em relação ao comentário a respeito dos mecanismos de ação da cardiomioplastia, gostaria de lembrar que nós também acreditamos que este procedimento atua, principalmente, sobre a mecânica da contração ventricular. Além da melhora da função sistólica do ventrículo esquerdo pela direta atuaçāo do enxerto muscular, a queda do "estresse" sistólico e diastólico daquela câmara e a conseqüente diminuição do consumo de oxigênio pelo miocárdio parecem ser alguns dos mecanismos principais da cardiomioplastia. Essas alteraçōes seriam, provavelmente, as responsáveis pela interrupção da progressão da miocardiopatia de base. Finalmente, podemos concluir que a cardiomioplastia ainda é um procedimento de caráter experimental, cujos resultados iniciais apontam para uma indicação mais precoce do que o transplante cardíaco, em pacientes que ainda mantenham algum grau de preservaçāo da função miocárdica. Os bons resultados apresentados nesse grupo especifico de pacientes, no entanto, abrem uma boa perspectiva para a utilizaçăo da cardiomioplastia como um procedimento paliativo no tratamento da insuficiência miocárdica. 